\title{
Bi-Criteria Optimization Technique in Stochastic System Maintenance Allocation Problem
}

\author{
Irfan Ali ${ }^{*}$, S. Suhaib Hasan \\ Department of Statistics \& Operations Research, Aligarh Muslim University, Aligarh, India \\ Email: ${ }^{*}$ irfii.ali@gmail.com
}

Received November 11, 2012; revised December 15, 2012; accepted December 28, 2012

\begin{abstract}
In this paper, the problem of optimum allocation of repairable and replaceable components in a system is formulated as a Bi-objective stochastic non linear programming problem. The system maintenance time and cost are random variable and has gamma and normal distribution respectively. A Bi-criteria optimization technique, weighted Tchebycheff is used to obtain the optimum allocation for a system. A numerical example is also presented to illustrate the computational details.
\end{abstract}

Keywords: Selective Maintenance; Weighted Tchebycheff Technique; Multi-Criteria Optimization; Stochastic Programming; Chance Constrained; Modified E-Model; System Reliability

\section{Introduction}

We consider a system which requires performing a sequence of identical production runs after every given (fixed) period. A production run in the system consists of several subsystems where each subsystem can work properly if at least one of its components is operational. The following assumptions are also made:

1) all the components can be repaired if deteriorated or failed;

2) all component states are independent.

We assume that the system comprises two types of subsystem. One is the type of subsystems in which the components are very sensitive to the functioning of the whole system and, therefore, on deterioration these should be replaced by new ones. Let these subsystems range from 1 to $s$. The other type of subsystems is those in which the components after deterioration can be re- paired and then replaced. Let such subsystems range from $s+1$ to $m$. In Figure 1 the Group $X$ consists of the $s$ subsystems with sensitive components which on failure are replaced by new ones and $Y$ the remaining $(m-s)$ subsystems in which the components can be repaired (see Ali et al. [1]).

Ideally, all the failed components in all the subsystem of Group X are replaced by new ones prior to the beginning of the next mission/ run. In a similar way, ideally all the failed components in subsystem of Group Y are repaired and then replaced prior to the beginning of the next mission/run. However, due to the constraints on the cost and time it may not be possible to repair and replace all the failed components in Groups X and Y. For this a mathematical programming frame-work is established for assisting decision-makers in determining the optimal subset of maintenance activities to perform prior to beginning of the next mission. This decision-making process

$\mathrm{X}$

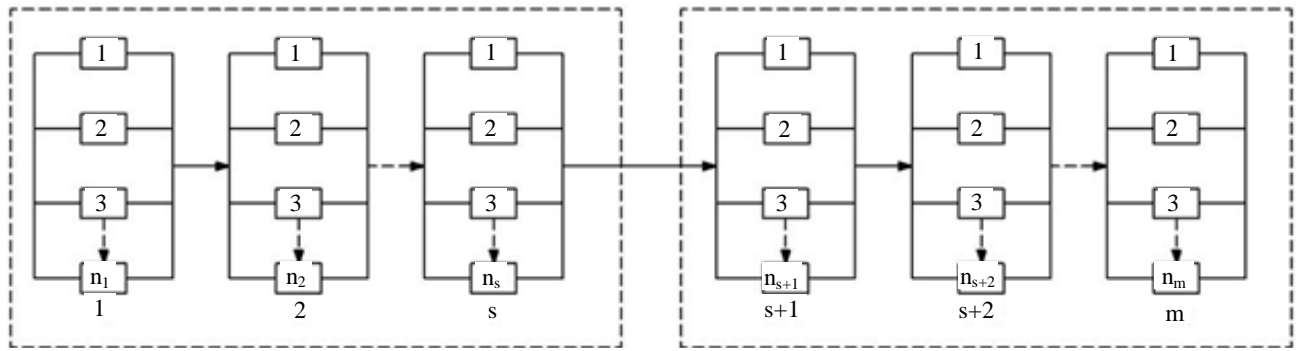

Figure 1. Parallel components in repairable and replaceable subsystem.

${ }^{*}$ Corresponding author. 
is referred to as selective maintenance. The selective maintenance models presented allow the decision-maker to consider limitations on maintenance time and budget, as well as the reliability of the system. Selective maintenance is an open research area that is consistent with the modern industrial objective of performing more intelligent and efficient maintenance.

For this let us suppose $a_{i}$ be the total failed components in the subsystems and $d_{i}$ be the number of components in the $i^{\text {th }}$ subsystem, which can be repaired and replaced prior to the beginning of the next mission (See Rice et al. [2]). Thus under the selective maintenance the number of components available for the next mission in the $i^{\text {th }}$ subsystem will be

$$
\left(n_{i}-k_{i}\right)+d_{i}, i=1,2, \cdots, m
$$

Therefore the reliability of the subsystems range from 1 to $S$ for a production run is given by

$$
R\left(d_{i}\right)=\left\{\prod_{i=1}^{s}\left[1-\left(1-r_{i}\right)^{n_{i}-a_{i}+d_{i}}\right]\right\}
$$

and the reliability of the subsystems range from $s+1$ to $m$ for a production run is given by

$$
R\left(d_{i}\right)=\left\{\prod_{i=S+1}^{m}\left[1-\left(1-r_{i}\right)^{n_{i}-a_{i}+d_{i}}\right]\right\}
$$

The maintenance time constraint for the system is given as

$$
\sum_{i=1}^{m} t_{i} d_{i} \leq T_{0}
$$

and the maintenance cost constraint for the system is given as

$$
\sum_{i=1}^{m} c_{i} d_{i} \leq C_{0}
$$

However, in the event the reliability of the subsystems of Groups $\mathrm{X}$ and $\mathrm{Y}$ time are of equally serious concern. Let us consider, for instance, the following multi-objective problem (please see the Equation (6) below).

Secondly, a Bi-objective programming problem in which time and the cost spent on system maintenance is minimized simultaneously for the required reliability $R_{i}^{*}\left(d_{i}\right)$ (say). The mathematical model of the problem is given as Equation (7) below.

Recently many authors have discussed the allocation problem of repairable components. Among them are Rice et al. [2], Schneider and Cassady [3], Rajaopalan and Cassady [4], Schneider et al. [5], Iyoob et al. [6], Ali et al. ([1,7-10]), Faisal and Ali [11] and many others.

In this paper, we have formulated stochastic system maintenance problem as a multi-objective programming

$$
\begin{aligned}
& \text { Maximize } R\left(d_{i}\right)=\left\{\prod_{i=1}^{s}\left[1-\left(1-r_{i}\right)^{n_{i}-a_{i}+d_{i}}\right]\right\} \\
& \text { and Maximize } R\left(d_{i}\right)=\left\{\prod_{i=S+1}^{m}\left[1-\left(1-r_{i}\right)^{n_{i}-a_{i}+d_{i}}\right]\right\} \\
& \text { Subject to } \sum_{i=1}^{m} t_{i} d_{i} \leq T_{0} \\
& \sum_{i=1}^{m} c_{i} d_{i} \leq C_{0} \\
& 0 \leq d_{i} \leq a_{i}, \forall d_{i} \text { areint eger } \\
& n_{i} \geq a_{i}, i=1,2, \cdots, m \\
& \text { Minimize } T=\sum_{i=1}^{m} t_{i} d_{i} \\
& \text { and Minimize } C=\sum_{i=1}^{m} c_{i} d_{i} C_{0} \\
& \text { Subject to }\left\{\prod_{i=1}^{s}\left[1-\left(1-r_{i}\right)^{n_{i}-a_{i}+d_{i}}\right]\right\} \geq R_{1}^{*}\left(d_{i}\right) \\
& \left\{\prod_{i=S+1}^{m}\left[1-\left(1-r_{i}\right)^{n_{i}-a_{i}+d_{i}}\right]\right\} \geq R_{2}^{*}\left(d_{i}\right) \\
& 0 \leq d_{i} \leq a_{i}, \forall d_{i} \text { are int eger } \\
& n_{i} \geq a_{i}, i=1,2, \cdots, m
\end{aligned}
$$


problem. We have discussed components repairable and replaceable time and cost as a random variable in the constraint and has Gamma and Normal distribution respectively. The Probabilistic constraints function is then converted into an equivalent deterministic non-linear programming form by using chance constrained programming.

\section{The Chance Constrained Programming}

In many practical situations the constraint Equations (iii) and (iv) are not fixed and taken as probabilistic. Thus the above problem (6) can be written in the following chance constrained programming form as Equation (8) below, where $p_{0}, 0 \leq p_{0} \leq 1$ is a specified probability.

In the above problem (8), let us assume that $t_{i}$ and $c_{i}$ are independently gamma and normally distributed random variables.

Let us assume that $t_{i}, i=1, \cdots, m$ are independent Gamma distributed random variables in the constraint 8 (iii), i.e., $t_{i} \sim G\left(\alpha_{i}, \beta_{i}\right)$.

Then the,

$$
\operatorname{Mean}\left(t_{i}\right)=\frac{\beta_{i}}{\alpha_{i}}, \text { Variance }\left(t_{i}\right)=\frac{\beta_{i}}{\alpha_{i}^{2}} .
$$

Now let

$$
f(d)=\sum_{i=1}^{m} t_{i} d_{i}
$$

Then mean is

$$
E\{f(d)\}=\sum_{i=1}^{m} d_{i} E\left(t_{i}\right)=\sum_{i=1}^{m} \frac{\beta_{i}}{\alpha_{i}} d_{i}
$$

Further, as $t_{i}$ are independently distributed, we have

$$
V\{f(d)\}=\sum_{i=1}^{m} t_{i}^{2} V\left(d_{i}\right)=\sum_{i=1}^{m} \frac{\beta_{i}}{\alpha_{i}^{2}} d_{i}
$$

Now the constraints 8 (iii) can be written as

$$
P\left(f(d) \leq T_{0}\right) \geq p_{0},
$$

Since number of components within the system are assumed to be large we have from Liapounoff's central limit theorem

$$
f(d) \sim N(E\{f(d)\}, V\{f(d)\}) .
$$

Thus (9) is equivalent to

$$
P\left\{\frac{f(d)-E\{f(d)\}}{\sqrt{V\{f(d)\}}} \leq \frac{T_{0}-E\{f(d)\}}{\sqrt{V\{f(d)\}}}\right\} \geq p_{0},
$$

where $\left[\frac{f(d)-E\{f(d)\}}{\sqrt{V\{f(d)\}}}\right]$ is a standard normal variate with mean zero and variance one. Thus the probability of realizing $\{f(d)\}$ less than or equal to $T_{0}$ can be written as

$$
P\left(f(d) \leq T_{0}\right)=\phi\left[\frac{T_{0}-E\{f(d)\}}{\sqrt{V\{f(d)\}}}\right],
$$

where $\phi(z)$ represents the cumulative density function of the standard normal variable evaluated at $Z$. If $K_{\alpha}$ represents the value of the standard normal variable at which $\phi\left(K_{\alpha}\right)=p_{0}$, then the constraint (10) can be written as

$$
\phi\left[\frac{T_{0}-E\{f(d)\}}{\sqrt{V\{f(d)\}}}\right] \geq \phi\left(K_{\alpha}\right) .
$$

The inequality will be satisfied only if

$$
\left[\frac{T_{0}-E\{f(d)\}}{\sqrt{V\{f(d)\}}}\right] \geq K_{\alpha},
$$

$$
\begin{aligned}
& \text { Maximize } R\left(d_{i}\right)=\left\{\prod_{i=1}^{s}\left[1-\left(1-r_{i}\right)^{n_{i}-a_{i}+d_{i}}\right]\right\} \\
& \text { and Maximize } R\left(d_{i}\right)=\left\{\prod_{i=S+1}^{m}\left[1-\left(1-r_{i}\right)^{n_{i}-a_{i}+d_{i}}\right]\right\} \\
& \text { Subject to } P\left(\sum_{i=1}^{m} t_{i} d_{i} \leq T_{0}\right) \geq p_{0} \\
& P\left(\sum_{i=1}^{m} c_{i} d_{i} \leq C_{0}\right) \geq p_{0} \\
& 0 \leq d_{i} \leq a_{i}, \forall d_{i} \text { are int eger } \\
& n_{i} \geq a_{i}, i=1,2, \cdots, m
\end{aligned}
$$


or equivalently,

$$
\frac{T_{0}-\sum_{i=1}^{m} \frac{\beta_{i}}{\alpha_{i}} d_{i}}{\sqrt{\sum_{i=1}^{m} \frac{\beta_{i}}{\alpha_{i}^{2}} d_{i}^{2}}} \geq K_{\alpha},
$$

Thus an equivalent deterministic constraint to the stochastic constraint is given by

$$
\begin{aligned}
& \left(\sum_{i=1}^{m} \frac{\beta_{i}}{\alpha_{i}} d_{i}\right) \\
& +K_{\alpha} \sqrt{\sum_{i=1}^{m} \frac{\beta_{i}}{\alpha_{i}^{2}} d_{i}^{2}} \leq T_{0}
\end{aligned}
$$

Now we consider the case when $c_{i}$ are independently normally distributed random variables in the constraint 8 (iv), i.e. $c_{i} \sim N\left(\mu_{i}, \sigma_{i}^{2}\right)$.

The constraint $P\left(f(c) \leq C_{0}\right) \geq p_{0}$, is equivalent to

$$
P\left\{\frac{f(c)-E\{f(c)\}}{\sqrt{V\{f(c)\}}} \leq \frac{C_{0}-E\{f(c)\}}{\sqrt{V\{f(c)\}}}\right\} \geq p_{0},
$$

Now in this case

$$
E\{f(c)\}=\sum_{i=1}^{m} d_{i} E\left(c_{i}\right)=\sum_{i=1}^{m} \mu_{i} d_{i}
$$

and

$$
V\{f(c)\}=\sum_{i=1}^{m} d_{i}^{2} V\left(c_{h}\right)=\sum_{i=1}^{m} \sigma_{i}^{2} d_{i}^{2}
$$

Therefore, the deterministic equivalent of 8 (iv) in this case is

$$
\left(\sum_{i=1}^{m} \mu_{i} d_{i}\right)+K_{\alpha} \sqrt{\sum_{i=1}^{m} \sigma_{i}^{2} d_{i}^{2}} \leq C_{0}
$$

The equivalent deterministic non-linear programming problem (8) to the stochastic programming problem is given by

$$
\begin{aligned}
& \text { Maximize } R\left(d_{i}\right)=\left\{\prod_{i=1}^{s}\left[1-\left(1-r_{i}\right)^{n_{i}-a_{i}+d_{i}}\right]\right\} \\
& \text { and Maximize } R\left(d_{i}\right)=\left\{\prod_{i=S+1}^{m}\left[1-\left(1-r_{i}\right)^{n_{i}-a_{i}+d_{i}}\right]\right\} \\
& \text { Subject to }\left(\sum_{i=1}^{m} \frac{\beta_{i}}{\alpha_{i}} d_{i}\right)+K_{\alpha} \sqrt{\sum_{i=1}^{m} \frac{\beta_{i}}{\alpha_{i}^{2}} d_{i}^{2}} \leq T_{0} \\
& \left(\sum_{i=1}^{m} \mu_{i} d_{i}\right)+K_{\alpha} \sqrt{\sum_{i=1}^{m} \sigma_{i}^{2} d_{i}^{2}} \leq C_{0} \\
& 0 \leq d_{i} \leq a_{i}, \forall d_{i} \text { are int eger } \\
& n_{i} \geq a_{i}, i=1,2, \cdots, m
\end{aligned}
$$

\section{Modified E-Model}

Consider the situations in which the time taken and cost spent on maintenance are not fixed and taken as prob- (i) abilistic in the objective function in Equations (i) and (ii). Thus the above problem (7) can be written in the following probabilistic objective function form as:

$$
\begin{aligned}
& \text { Minimize } T=p\left(\sum_{i=1}^{m} t_{i} d_{i}\right) \\
& \text { and Minimize } C=p\left(\sum_{i=1}^{m} c_{i} d_{i}\right) \\
& \text { Subject to }\left\{\prod_{i=1}^{s}\left[1-\left(1-r_{i}\right)^{n_{i}-a_{i}+d_{i}}\right]\right\} \times\left\{\prod_{i=S+1}^{m}\left[1-\left(1-r_{i}\right)^{n_{i}-a_{i}+d_{i}}\right]\right\} \geq R^{*}\left(d_{i}\right) \\
& 0 \leq d_{i} \leq a_{i}, \forall d_{i} \text { areint eger } \\
& n_{i} \geq a_{i}, i=1,2, \cdots, m
\end{aligned}
$$


Using Modified E-model technique, the problem (16) is formulated as

$$
\begin{aligned}
& \text { Minimize } T=k_{1}\left(\sum_{i=1}^{m} \frac{\beta_{i}}{\alpha_{i}} d_{i}\right)+k_{2} \sqrt{\sum_{i=1}^{m} \frac{\beta_{i}}{\alpha_{i}^{2}} d_{i}^{2}} \\
& \text { and Minimize } C=k_{1}\left(\sum_{i=1}^{m} \mu_{i} d_{i}\right)+k_{2} \sqrt{\sum_{i=1}^{m} \sigma_{i}^{2} d_{i}^{2}} \\
& \text { Subject to }\left\{\prod_{i=1}^{s}\left[1-\left(1-r_{i}\right)^{n_{i}-a_{i}+d_{i}}\right]\right\} \times\left\{\prod_{i=S+1}^{m}\left[1-\left(1-r_{i}\right)^{n_{i}-a_{i}+d_{i}}\right]\right\} \geq R^{*}\left(d_{i}\right) \\
& 0 \leq d_{i} \leq a_{i}, \forall d_{i} \text { are int eger } \\
& n_{i} \geq a_{i}, i=1,2, \cdots, m
\end{aligned}
$$

where $k_{1}$ and $k_{2}$ are non-negative constants, and their values show the relative importance of the expectation and the variance. Some authors suggest that $k_{1}+k_{2}=1$, see Rao [12].

The two others Bi-objective programming models in different prospects for the decision-makers are

\section{Model 1:}

$$
\begin{aligned}
& \text { Minimize } T=k_{1}\left(\sum_{i=1}^{m} \frac{\beta_{i}}{\alpha_{i}} d_{i}\right)+k_{2} \sqrt{\sum_{i=1}^{m} \frac{\beta_{i}}{\alpha_{i}^{2}} d_{i}^{2}} \\
& \text { and Maximize } R_{2}\left(d_{i}\right)=\left\{\prod_{i=S+1}^{m}\left[1-\left(1-r_{i}\right)^{n_{i}-a_{i}+d_{i}}\right]\right\} \\
& \text { Subject to }\left(\sum_{i=1}^{m} \mu_{i} d_{i}\right)+K_{\alpha} \sqrt{\sum_{i=1}^{m} \sigma_{i}^{2} d_{i}^{2}} \leq C_{0} \\
& \left\{\prod_{i=1}^{s}\left[1-\left(1-r_{i}\right)^{n_{i}-a_{i}+d_{i}}\right]\right\} \times\left\{\prod_{i=S+1}^{m}\left[1-\left(1-r_{i}\right)^{n_{i}-a_{i}+d_{i}}\right]\right\} \geq R^{*}\left(d_{i}\right) \\
& 0 \leq d_{i} \leq a_{i}, \forall d_{i} \text { are int eger } \\
& n_{i} \geq a_{i}, i=1,2, \cdots, m
\end{aligned}
$$

Model 2:

$$
\begin{aligned}
& \text { Minimize } C=k_{1}\left(\sum_{i=1}^{m} \mu_{i} d_{i}\right)+k_{2} \sqrt{\sum_{i=1}^{m} \sigma_{i}^{2} d_{i}^{2}} \\
& \text { and Maximize } R_{1}\left(d_{i}\right)=\left\{\prod_{i=1}^{s}\left[1-\left(1-r_{i}\right)^{n_{i}-a_{i}+d_{i}}\right]\right\} \\
& \text { Subject to }\left(\sum_{i=1}^{m} \frac{\beta_{i}}{\alpha_{i}} d_{i}\right)+K_{\alpha} \sqrt{\sum_{i=1}^{m} \frac{\beta_{i}}{\alpha_{i}^{2}} d_{i}^{2}} \leq T_{0} \\
& \left\{\prod_{i=1}^{s}\left[1-\left(1-r_{i}\right)^{n_{i}-a_{i}+d_{i}}\right]\right\} \times\left\{\prod_{i=S+1}^{m}\left[1-\left(1-r_{i}\right)^{n_{i}-a_{i}+d_{i}}\right]\right\} \geq R^{*}\left(d_{i}\right) \\
& 0 \leq d_{i} \leq a_{i}, \forall d_{i} \text { are int eger } \\
& n_{i} \geq a_{i}, i=1,2, \cdots, m
\end{aligned}
$$

\section{A Multi-Criteria Weighted Tchebycheff Optimization Technique}

Let us consider a multi-objective programming prob- lem

$$
\left.\begin{array}{l}
\text { Min } f(x)=f_{1}(x), f_{2}(x), \cdots, f_{k}(x) \\
\text { Subject to } x \in S
\end{array}\right\}
$$


assumed to have $k(k \geq 2)$ competing objective functions $\left(f_{i}: \Re^{n} \rightarrow \mathfrak{R}\right)$ that are to be minimized simultaneously. The following definitions illustrate the concepts of efficient and weakly efficient decision vectors.

Definition: A decision vector $x^{*} \in X$ is efficient (Pareto optimal) for multi-objective programming problem if there does not exist a $x \in X, x \neq x^{*}$ such that $f_{i}(x) \leq f_{i}\left(x^{*}\right)$ for $i=1,2, \cdots, k$ with strict inequality holding for at least one index $i . \quad\left(x^{*} \in X\right.$ is efficient, $f\left(x^{*}\right)$ is non-dominated).

Definition: A decision vector $x^{*} \in X$ is weakly efficient (weakly Pareto optimal) for multi-objective programming problem if there does not exist a $x \in X$, $x \neq x^{*}$ such that $f_{i}(x)<f_{i}\left(x^{*}\right)$ for $i=1,2, \cdots, k$. ( $x^{*} \in X$ is weakly efficient, $f\left(x^{*}\right)$ is weakly nondominated).

There are several metrics that are found in the literature related to multi-objective programming problem. If $\xi_{i}$ is the reference point and the ideal point,

$$
\xi_{i}=\operatorname{Min}_{x \in X} f_{i}(x),
$$

is used as the reference point, the general weighted $L_{p}$-metric $(1 \leq p \leq \infty)$ is defined as

$$
\left.\begin{array}{l}
\operatorname{Min}\left(\sum_{i=1}^{k} w_{i}\left|f_{i}(x)-\xi_{i}\right|^{p}\right)^{1 / p} \\
\text { Subject to } x \in X
\end{array}\right\}
$$

We assume that $w_{i} \geq 0, \forall i=1,2, \cdots, k$ and $\sum_{i=1}^{k} w_{i}=1$, where the $w_{i}$ 's are weighting coefficients provided by the decision maker reflecting the relative importance. If $p=\infty$, problem (21) reduces to a "weighted Tchebycheff Technique” (see Bowman [13]).

$$
\left.\begin{array}{l}
\operatorname{Min} \underset{i=1,2, \cdots, k}{\operatorname{Max}}\left[w_{i}\left|f_{i}(x)-\xi_{i}\right|\right] \\
\text { Subject to } x \in X
\end{array}\right\}
$$

If the reference point is the global optimal solution of $f_{i}(x)$, then the absolute value signs in problem (22) can be removed (see Miettinen [14]) yielding

$$
\left.\begin{array}{l}
\operatorname{Min} \underset{i=1,2, \cdots, k}{\operatorname{Max}}\left[w_{i}\left(f_{i}(x)-\xi_{i}\right)\right] \\
\text { Subject to } x \in X
\end{array}\right\}
$$

Miettinen [14] also showed that if the objectives and constraints are differentiable form of problem (23) can be defined as

$$
\begin{aligned}
& \text { Min } \delta \\
& \text { Subject to } \left.\delta \geq\left\{w_{i}\left(f_{i}(x)-\xi_{i}\right)\right\}, \forall i, x \in X\right\}
\end{aligned}
$$

The solution of problem (24) is guaranteed weekly non-dominated for positive weights and at least one non-dominated solution is also guaranteed. If the solution is unique, then it is non-dominated, however if it is not unique, then it might be weakly non-dominated (see Wierzbicki [15].

The two objective functions in the Equation (15) are to be maximizing the total reliability of replaceable components of Group X and the reliability of repairable components of Group Y. We have convert the following maximizing problem into minimization problem using the property $\operatorname{Max} Z=\operatorname{Min}(-Z)$. Therefore the problem defined in Equation (15) is converted into a two criterion minimization problem: $\operatorname{Min}-\left\{Z_{1}, Z_{2}\right\}$ subject to the constraints (see Khasawneh et al. [16]). Now the efficient solution is obtained by using the weighted Tchebycheff technique

Minimize $\delta$

$$
\begin{aligned}
& \text { Subject to } \delta \geq w_{1}\left\{\left\{-\prod_{i=1}^{s}\left[1-\left(1-r_{i}\right)^{n_{i}-a_{i}+d_{i}}\right]\right\}-\xi_{1}\right\} \\
& \delta \geq w_{2}\left\{\left\{\prod_{i=S+1}^{m}\left[1-\left(1-r_{i}\right)^{n_{i}-a_{i}+d_{i}}\right]\right\}-\xi_{2}\right\} \\
& \left(\sum_{i=1}^{m} \frac{\beta_{i}}{\alpha_{i}} t_{i}\right)+K_{\alpha} \sqrt{\sum_{i=1}^{m} \frac{\beta_{i}}{\alpha_{i}^{2}} d_{i}^{2}} \leq T_{0} \\
& \left(\sum_{i=1}^{m} \mu_{i} d_{i}\right)+K_{\alpha} \sqrt{\sum_{i=1}^{m} \sigma_{i}^{2} d_{i}^{2}} \leq C_{0} \\
& w_{1}+w_{2}=1, w_{1}, w_{2} \geq 0 \\
& 0 \leq d_{i} \leq a_{i}, \forall d_{i} \text { are int eger } \\
& n_{i} \geq a_{i}, i=1,2, \cdots, m
\end{aligned}
$$

In practice, $\xi_{i}$ can be defined as the minimum individual values of the following problems: 


$$
\begin{aligned}
& \xi_{1}=\operatorname{Min}\left(-R\left(d_{i}\right)\right)=-\left\{\prod_{i=1}^{s}\left[1-\left(1-r_{i}\right)^{n_{i}-a_{i}+d_{i}}\right]\right\} \\
& \text { Subject to }\left(\sum_{i=1}^{m} \frac{\beta_{i}}{\alpha_{i}} t_{i}\right)+K_{\alpha} \sqrt{\sum_{i=1}^{m} \frac{\beta_{i}}{\alpha_{i}^{2}} d_{i}^{2}} \leq T_{0} \\
& \left(\sum_{i=1}^{m} \mu_{i} d_{i}\right)+K_{\alpha} \sqrt{\sum_{i=1}^{m} \sigma_{i}^{2} d_{i}^{2}} \leq C_{0} \\
& 0 \leq d_{i} \leq a_{i}, \forall d_{i} \text { are int eger } \\
& n_{i} \geq a_{i}, i=1,2, \cdots, m
\end{aligned}
$$

and similarly

$$
\begin{aligned}
& \xi_{2}=\operatorname{Min}\left(-R\left(d_{i}\right)\right)=-\left\{\prod_{i=s+1}^{m}\left[1-\left(1-r_{i}\right)^{n_{i}-a_{i}+d_{i}}\right]\right\} \\
& \text { Subject to }\left(\sum_{i=1}^{m} \frac{\beta_{i}}{\alpha_{i}} t_{i}\right)+K_{\alpha} \sqrt{\sum_{i=1}^{m} \frac{\beta_{i}}{\alpha_{i}^{2}} d_{i}^{2}} \leq T_{0} \\
& \left(\sum_{i=1}^{m} \mu_{i} d_{i}\right)+K_{\alpha} \sqrt{\sum_{i=1}^{m} \sigma_{i}^{2} d_{i}^{2}} \leq C_{0} \\
& 0 \leq d_{i} \leq a_{i}, \forall d_{i} \text { are int eger } \\
& n_{i} \geq a_{i}, i=1,2, \cdots, m
\end{aligned}
$$

In similar way, the problem defined in Equation (17) is also a two criterion minimization problem: $\operatorname{Min}\{T, C\}$ subject to the constraints. Now the efficient solution is obtained by using the weighted Tchebycheff technique
Minimize $\delta$

Subject to $\delta \geq w_{1}\left\{\left\{k_{1}\left(\sum_{i=1}^{m} \frac{\beta_{i}}{\alpha_{i}} t_{i}\right)+k_{2} \sqrt{\sum_{i=1}^{m} \frac{\beta_{i}}{\alpha_{i}^{2}} d_{i}^{2}}\right\}-\xi_{1}\right\}$

$\delta \geq w_{2}\left\{k_{1}\left(\sum_{i=1}^{m} \mu_{i} d_{i}\right)+k_{2} \sqrt{\sum_{i=1}^{m} \sigma_{i}^{2} d_{i}^{2}}-\xi_{2}\right\}$

$\left\{\prod_{i=1}^{s}\left[1-\left(1-r_{i}\right)^{n_{i}-a_{i}+d_{i}}\right]\right\} \times\left\{\prod_{i=S+1}^{m}\left[1-\left(1-r_{i}\right)^{n_{i}-a_{i}+d_{i}}\right]\right\} \geq R^{*}\left(d_{i}\right)$

$w_{1}+w_{2}=1, w_{1}, w_{2} \geq 0$

$0 \leq d_{i} \leq a_{i}, \forall d_{i}$ are int eger

$n_{i} \geq a_{i}, i=1,2, \cdots, m$ (i) 
The values of $\xi_{i}$ can be defined as the minimum individual values of the following problems:

$$
\begin{aligned}
& \xi_{1}=\operatorname{Min} T=k_{1}\left(\sum_{i=1}^{m} \frac{\beta_{i}}{\alpha_{i}} d_{i}\right)+k_{2} \sqrt{\sum_{i=1}^{m} \frac{\beta_{i}}{\alpha_{i}^{2}} d_{i}^{2}} \\
& \text { Subject to }\left\{\prod_{i=1}^{s}\left[1-\left(1-r_{i}\right)^{n_{i}-a_{i}+d_{i}}\right]\right\} \times\left\{\prod_{i=S+1}^{m}\left[1-\left(1-r_{i}\right)^{n_{i}-a_{i}+d_{i}}\right]\right\} \geq R^{*}\left(d_{i}\right) \\
& 0 \leq d_{i} \leq a_{i}, \forall d_{i} \text { are int eger } \\
& n_{i} \geq a_{i}, i=1,2, \cdots, m
\end{aligned}
$$

and similarly

$$
\begin{aligned}
& \xi_{2}=\operatorname{Min} C=k_{1}\left(\sum_{i=1}^{m} \mu_{i} d_{i}\right)+k_{2} \sqrt{\sum_{i=1}^{m} \sigma_{i}^{2} d_{i}^{2}} \\
& \text { Subject to }\left\{\prod_{i=1}^{s}\left[1-\left(1-r_{i}\right)^{n_{i}-a_{i}+d_{i}}\right]\right\} \times\left\{\prod_{i=S+1}^{m}\left[1-\left(1-r_{i}\right)^{n_{i}-a_{i}+d_{i}}\right]\right\} \geq R^{*}\left(d_{i}\right) \\
& 0 \leq d_{i} \leq a_{i}, \forall d_{i} \text { are int eger } \\
& n_{i} \geq a_{i}, i=1,2, \cdots, m
\end{aligned}
$$

Now the problem defined in Model 1; Equation (18) is also a two criterion minimization problem:

$\operatorname{Min}\left\{T-R_{2}\left(d_{i}\right)\right\}$ subject to the constraints. Now the

\section{Minimize $\delta$}

$$
\begin{aligned}
& \text { Subject to } \delta \geq w_{1}\left\{\left\{k_{1}\left(\sum_{i=1}^{m} \frac{\beta_{i}}{\alpha_{i}} t_{i}\right)+k_{2} \sqrt{\sum_{i=1}^{m} \frac{\beta_{i}}{\alpha_{i}^{2}} d_{i}^{2}}\right\}-\xi_{1}\right\} \\
& \delta \geq w_{2}\left\{\left\{\prod_{i=S+1}^{m}\left[1-\left(1-r_{i}\right)^{n_{i}-a_{i}+d_{i}}\right]\right\}-\xi_{2}\right\} \\
& \left\{\prod_{i=1}^{s}\left[1-\left(1-r_{i}\right)^{n_{i}-a_{i}+d_{i}}\right]\right\} \times\left\{\prod_{i=S+1}^{m}\left[1-\left(1-r_{i}\right)^{n_{i}-a_{i}+d_{i}}\right]\right\} \geq R^{*}\left(d_{i}\right) \\
& \left(\sum_{i=1}^{m} \mu_{i} d_{i}\right)+K_{\alpha} \sqrt{\sum_{i=1}^{m} \sigma_{i}^{2} d_{i}^{2}} \leq C_{0} \\
& w_{1}+w_{2}=1, w_{1}, w_{2} \geq 0 \\
& 0 \leq d_{i} \leq a_{i}, \forall d_{i} \text { are int eger } \\
& n_{i} \geq a_{i}, i=1,2, \cdots, m
\end{aligned}
$$

efficient solution is obtained by using the weighted Tchebycheff technique

\section{(i)}

The values of $\xi_{1}$ and $\xi_{2}$ is the minimum individual values obtained as

$$
\begin{aligned}
\xi_{1} & =\text { Minimize } T \\
& =k_{1}\left(\sum_{i=1}^{m} \frac{\beta_{i}}{\alpha_{i}} d_{i}\right)+k_{2} \sqrt{\sum_{i=1}^{m} \frac{\beta_{i}}{\alpha_{i}^{2}} d_{i}^{2}}
\end{aligned}
$$

subject to the (iii) to (vi) of Equation (18) 
Minimize $\delta$

$$
\begin{aligned}
& \text { Subject to } \delta \geq w_{1}\left\{k_{1}\left(\sum_{i=1}^{m} \mu_{i} d_{i}\right)+k_{2} \sqrt{\sum_{i=1}^{m} \sigma_{i}^{2} d_{i}^{2}}-\xi_{1}\right\} \\
& \delta \geq w_{2}\left\{\left\{\prod_{i=1}^{s}\left[1-\left(1-r_{i}\right)^{n_{i}-a_{i}+d_{i}}\right]\right\}-\xi_{2}\right\} \\
& \left\{\prod_{i=1}^{s}\left[1-\left(1-r_{i}\right)^{n_{i}-a_{i}+d_{i}}\right]\right\} \times\left\{\prod_{i=S+1}^{m}\left[1-\left(1-r_{i}\right)^{n_{i}-a_{i}+d_{i}}\right]\right\} \geq R^{*}\left(d_{i}\right) \\
& \left(\sum_{i=1}^{m} \frac{\beta_{i}}{\alpha_{i}} t_{i}\right)+K_{\alpha} \sqrt{\sum_{i=1}^{m} \frac{\beta_{i}}{\alpha_{i}^{2}} d_{i}^{2}} \leq T_{0} \\
& w_{1}+w_{2}=1, w_{1}, w_{2} \geq 0 \\
& 0 \leq d_{i} \leq a_{i}, \forall d_{i} \text { are int eger } \\
& n_{i} \geq a_{i}, i=1,2, \cdots, m
\end{aligned}
$$

where the values of $\xi_{1}$ and $\xi_{2}$ is the minimum individual values obtained as

$$
\xi_{1}=\text { Minimize } C=k_{1}\left(\sum_{i=1}^{m} \mu_{i} d_{i}\right)+k_{2} \sqrt{\sum_{i=1}^{m} \sigma_{i}^{2} d_{i}^{2}}
$$

subject to the (iii) to (vi) of Equation (19)

$$
\xi_{2}=\operatorname{Min} R_{1}\left(d_{i}\right)=-\left\{\prod_{i=1}^{s}\left[1-\left(1-r_{i}\right)^{n_{i}-a_{i}+d_{i}}\right]\right\}
$$

subject to the (iii) to (vi) of Equation (19).

\section{Numerical Illustrations}

Consider a system having the Group X consisting of 3 subsystems and also the Group Y consisting of 4 subsystems. The available time between two missions for repairing and replacing is 150 time units. The available cost of maintenance for repairing and replacing for the next mission is 860 units. For simplicity we have considered in the above numerical illustration: the reliability of each component in a subsystem is same, cost spent and time taken on replacing and repairing each component within a subsystem are same. The remaining parameters for the various subsystems are given in Table $\mathbf{1}$

\subsection{Solution of Chance Constrained Programming by Using Weighted Tchebycheff Technique}

Before applying the Weighted Tchebycheff Technique firstly we find the individual optimum values $\xi_{i}=\left(\xi_{1}, \xi_{2}\right)$. For the values given in Table 1, the SNLPP (26) for the first optimum value is

$$
\left.\begin{array}{l}
\xi_{1}=\operatorname{Min}\left(-R_{1}\left(d_{i}\right)\right)=-\left\{\left[1-(1-0.8)^{\left(3+d_{1}\right)}\right] \times\left[1-(1-0.75)^{\left(2+d_{2}\right)}\right] \times\left[1-(1-0.8)^{\left(4+d_{3}\right)}\right]\right\}, \\
\text { Subject to }\left[2 d_{1}+3 d_{2}+d_{3}+20 d_{4}+28 d_{5}+22 d_{6}+22 d_{7}\right] \\
+2.99 \sqrt{0.33 d_{1}^{2}+0.60 d_{2}^{2}+0.10 d_{3}^{2}+2.86 d_{4}^{2}+3.11 d_{5}^{2}+1.83 d_{6}^{2}+2.2 d_{7}^{2}} \leq 150 \\
{\left[120 d_{1}+110 d_{2}+120 d_{3}+40 d_{4}+30 d_{5}+45 d_{6}+65 d_{7}\right]} \\
+2.99 \sqrt{13 d_{1}^{2}+10 d_{2}^{2}+15 d_{3}^{2}+4 d_{4}^{2}+3 d_{5}^{2}+5 d_{6}^{2}+6 d_{7}^{2}} \leq 860 \\
0 \leq d_{i} \leq a_{i}, \forall d_{i} \text { are int eger } n_{i} \geq a_{i}, i=1,2, \cdots, 7 .
\end{array}\right\}
$$

The optimal solution of (33) provided by LINGO is

$$
d_{1}^{*}=2, d_{2}^{*}=3, d_{3}^{*}=2, d_{4}^{*}=0, d_{5}^{*}=0, d_{6}^{*}=0, d_{7}^{*}=0
$$

with the value of objective function as

$$
R_{1}\left(d_{i j}\right)=0.9986398 .
$$

Similarly using (27) the SNLPP for the second optimum values 
Table 1. The number of failed components and the respective cost and time etc. in the various subsystems.

\begin{tabular}{lccccccc}
\hline & Group X (Replaced) & \multicolumn{5}{c}{ Group Y (Repair) } \\
\hline Subsystem & $\mathbf{1}$ & $\mathbf{2}$ & $\mathbf{3}$ & $\mathbf{4}$ & $\mathbf{5}$ & $\mathbf{6}$ & $\mathbf{7}$ \\
\hline$n_{i}$ & 6 & 5 & 10 & 7 & 9 & 12 & 10 \\
$r_{i}$ & 0.8 & 0.75 & 0.8 & 0.8 & 0.75 & 0.8 & 0.7 \\
$\beta_{i}$ & 12 & 15 & 10 & 140 & 252 & 264 & 220 \\
$\alpha_{i}$ & 6 & 5 & 10 & 7 & 9 & 12 & 10 \\
$E\left(t_{i}\right)=\frac{\beta_{i}}{\alpha_{i}}$ & 2 & 3 & 1 & 20 & 28 & 22 & 22 \\
$V\left(t_{i}\right)=\frac{\beta_{i}}{\alpha_{i}^{2}}$ & 0.33 & 0.60 & 0.10 & 2.86 & 3.11 & 1.83 & 2.2 \\
$\bar{c}_{i}$ & 120 & 110 & 120 & 40 & 30 & 45 & 65 \\
$\sigma_{c_{i}}^{2}$ & 13 & 10 & 15 & 4 & 3 & 5 & 6 \\
$a_{i}$ & 3 & 3 & 6 & 5 & 7 & 9 & 7 \\
\hline
\end{tabular}

$\xi_{2}=\operatorname{Min}\left(-R_{2}\left(d_{i}\right)\right)=-\left\{\left[1-(1-0.8)^{\left(2+d_{1}\right)}\right] \times\left[1-(1-0.75)^{\left(2+d_{2}\right)}\right] \times\left[1-(1-0.8)^{\left(3+d_{3}\right)}\right] \times\left[1-(1-0.70)^{\left(3+d_{2}\right)}\right]\right\}$,

Subject to $\left[2 d_{1}+3 d_{2}+d_{3}+20 d_{4}+28 d_{5}+22 d_{6}+22 d_{7}\right]$

$+2.99 \sqrt{0.33 d_{1}^{2}+0.60 d_{2}^{2}+0.10 d_{3}^{2}+2.86 d_{4}^{2}+3.11 d_{5}^{2}+1.83 d_{6}^{2}+2.2 d_{7}^{2}} \leq 150$

$\left[120 d_{1}+110 d_{2}+120 d_{3}+40 d_{4}+30 d_{5}+45 d_{6}+65 d_{7}\right]+2.99 \sqrt{13 d_{1}^{2}+10 d_{2}^{2}+15 d_{3}^{2}+4 d_{4}^{2}+3 d_{5}^{2}+5 d_{6}^{2}+6 d_{7}^{2}} \leq 860$

$0 \leq d_{i} \leq a_{i}, \forall d_{i}$ are int eger $n_{i} \geq a_{i}, i=1,2, \cdots, 7$.

The optimal solution of (34) provided by LINGO is

$$
d_{1}^{*}=0, d_{2}^{*}=0, d_{3}^{*}=0, d_{4}^{*}=2, d_{5}^{*}=1, d_{6}^{*}=1, d_{7}^{*}=2
$$

with the value of objective function as

$$
R_{2}\left(d_{i j}\right)=0.9788431 \text {. }
$$

From the Equations (33) and (34) the optimum values $\xi=(-0.9986398,-0.9788431)$. For simplicity we assumed that the reliability of both the Groups $\mathrm{X}$ and $\mathrm{Y}$ subsystems are equally important, that is $w_{1}=w_{2}=0.5$. For the values given in Table 1, the SNLPP (25) efficient solution is obtained by using the weighted Tchebycheff Technique

Minimize $\delta$

Subject to $\delta \geq w_{1}\left\{-\left\{\left[1-(1-0.8)^{\left(3+d_{1}\right)}\right] \times\left[1-(1-0.75)^{\left(2+d_{2}\right)}\right] \times\left[1-(1-0.8)^{\left(4+d_{3}\right)}\right]\right\}-(-0.9983842)\right\}$

$\delta \geq w_{2}\left\{-\left\{\left[1-(1-0.8)^{\left(2+d_{4}\right)}\right] \times\left[1-(1-0.75)^{\left(2+d_{5}\right)}\right] \times\left[1-(1-0.8)^{\left(3+d_{6}\right)}\right] \times\left[1-(1-0.7)^{\left(3+d_{7}\right)}\right]\right\}-(-0.9994604)\right\}$

$\left[2 d_{1}+3 d_{2}+d_{3}+20 d_{4}+28 d_{5}+22 d_{6}+22 d_{7}\right]$

$+2.99 \sqrt{0.15 d_{1}^{2}+0.18 d_{2}^{2}+0.10 d_{3}^{2}+0.35 d_{4}^{2}+0.40 d_{5}^{2}+0.50 d_{6}^{2}+0.60 d_{7}^{2}} \leq 150$

$\left[120 d_{1}+110 d_{2}+120 d_{3}+40 d_{4}+30 d_{5}+45 d_{6}+65 d_{7}\right]+2.99 \sqrt{10 d_{1}^{2}+8 d_{2}^{2}+15 d_{3}^{2}+8 d_{4}^{2}+5 d_{5}^{2}+7 d_{6}^{2}+9 d_{7}^{2}} \leq 860$

$0 \leq d_{i} \leq a_{i}, \forall d_{i}$ are int eger, $w_{1}+w_{2}=1, w_{1}, w_{2} \geq 0, n_{i} \geq a_{i}, i=1,2, \cdots, 7$. 
The optimum allocation under the Weighted Tchebycheff Technique

$$
d_{\text {Tcheb }}^{*}=\left(\underline{d}_{1}^{*}, d_{2}^{*}, \underline{d}_{3}^{*}, \underline{d}_{4}^{*}, \underline{d}_{5}^{*}, \underline{d}_{6}^{*}, \underline{d}_{7}^{*}\right)
$$

is obtained as

$$
d_{1}^{*}=2, d_{2}^{*}=3, d_{3}^{*}=0, d_{4}^{*}=2, d_{5}^{*}=2, d_{6}^{*}=0, d_{7}^{*}=1 .
$$

The corresponding value of objective function is
0.00076

\subsection{Solution of Modified E-Model by Using Weighted Tchebycheff Technique}

The individual optimum values $\xi_{i}=\left(\xi_{1}, \xi_{2}\right)$. For the values given in Table $\mathbf{1}$ and for simplicity take $k_{1}=k_{2}=0.5$ then the SNLPP (29) for the first optimum value is

$$
\left.\begin{array}{l}
\begin{array}{rl}
\xi_{1}=\operatorname{Min} T & =k_{1}\left[2 d_{1}+3 d_{2}+d_{3}+20 d_{4}+28 d_{5}+22 d_{6}+22 d_{7}\right] \\
+ & k_{2} \sqrt{0.33 d_{1}^{2}+0.60 d_{2}^{2}+0.10 d_{3}^{2}+2.86 d_{4}^{2}+3.11 d_{5}^{2}+1.83 d_{6}^{2}+2.2 d_{7}^{2}}
\end{array} \\
\text { Subject to }\left\{\left[1-(1-0.8)^{\left(3+d_{1}\right)}\right] \times\left[1-(1-0.75)^{\left(2+d_{2}\right)}\right] \times\left[1-(1-0.8)^{\left(4+d_{3}\right)}\right]\right\} \\
\quad \times\left\{\left[1-(1-0.8)^{\left(2+d_{1}\right)}\right] \times\left[1-(1-0.75)^{\left(2+d_{2}\right)}\right] \times\left[1-(1-0.8)^{\left(3+d_{3}\right)}\right] \times\left[1-(1-0.70)^{\left(3+d_{2}\right)}\right]\right\} \geq 0.99
\end{array}\right\}
$$

And the SNLPP (30) for the second optimum value

$$
\left.\begin{array}{l}
\begin{array}{rl}
\xi_{2}=\text { MinC } & =k_{1}\left[120 d_{1}+110 d_{2}+120 d_{3}+40 d_{4}+30 d_{5}+45 d_{6}+65 d_{7}\right] \\
+ & k_{2} \sqrt{10 d_{1}^{2}+8 d_{2}^{2}+15 d_{3}^{2}+8 d_{4}^{2}+5 d_{5}^{2}+7 d_{6}^{2}+9 d_{7}^{2}}
\end{array} \\
\text { Subject to }\left\{\left[1-(1-0.8)^{\left(3+d_{1}\right)}\right] \times\left[1-(1-0.75)^{\left(2+d_{2}\right)}\right] \times\left[1-(1-0.8)^{\left(4+d_{3}\right)}\right]\right\} \\
\quad \times\left\{\left[1-(1-0.8)^{\left(2+d_{1}\right)}\right] \times\left[1-(1-0.75)^{\left(2+d_{2}\right)}\right] \times\left[1-(1-0.8)^{\left(3+d_{3}\right)}\right] \times\left[1-(1-0.70)^{\left(3+d_{2}\right)}\right]\right\} \geq 0.99
\end{array}\right\}
$$

From the Equations (36) and (37) the optimum values $\xi=(101.73,418.40)$.

For simplicity we assumed that the maintenance time taken and cost spent for both the Groups X and Y sub- systems are equally important, that is $w_{1}=w_{2}=0.5$. For the values given in Table 1, the SNLPP (28) efficient solution is obtained by using the weighted Tchebycheff Technique

Minimize $\delta$

Subject to $\delta \geq w_{1}\left\{\left\{0.5\left[2 d_{1}+3 d_{2}+d_{3}+20 d_{4}+28 d_{5}+22 d_{6}+22 d_{7}\right]\right.\right.$

$$
\begin{aligned}
& \left.\left.+0.5 \sqrt{0.33 d_{1}^{2}+0.60 d_{2}^{2}+0.10 d_{3}^{2}+2.86 d_{4}^{2}+3.11 d_{5}^{2}+1.83 d_{6}^{2}+2.2 d_{7}^{2}}\right\}-101.73\right\} \\
\delta \geq & w_{2}\left\{-\left\{0.5\left[120 d_{1}+110 d_{2}+120 d_{3}+40 d_{4}+30 d_{5}+45 d_{6}+65 d_{7}\right]\right.\right. \\
& \left.\left.+0.5 \sqrt{10 d_{1}^{2}+8 d_{2}^{2}+15 d_{3}^{2}+8 d_{4}^{2}+5 d_{5}^{2}+7 d_{6}^{2}+9 d_{7}^{2}}\right\}-418.40\right\}
\end{aligned}
$$

Subject to $\left\{\left[1-(1-0.8)^{\left(3+d_{1}\right)}\right] \times\left[1-(1-0.75)^{\left(2+d_{2}\right)}\right] \times\left[1-(1-0.8)^{\left(4+d_{3}\right)}\right]\right\}$

$$
\times\left\{\left[1-(1-0.8)^{\left(2+d_{1}\right)}\right] \times\left[1-(1-0.75)^{\left(2+d_{2}\right)}\right] \times\left[1-(1-0.8)^{\left(3+d_{3}\right)}\right] \times\left[1-(1-0.70)^{\left(3+d_{2}\right)}\right]\right\} \geq 0.99
$$

$0 \leq d_{i} \leq a_{i}, \forall d_{i}$ are int eger, $n_{i} \geq a_{i}, i=1,2, \cdots, 7$.

The optimum allocation under the Weighted Tcheby- cheff Technique 


$$
d_{\text {Tcheb }}^{*}=\left(\underline{d}_{1}^{*}, d_{2}^{*}, \underline{d}_{3}^{*}, \underline{d}_{4}^{*}, \underline{d}_{5}^{*}, \underline{d}_{6}^{*}, \underline{d}_{7}^{*}\right)
$$

is obtained as

$$
d_{1}^{*}=1, d_{2}^{*}=3, d_{3}^{*}=0, d_{4}^{*}=3, d_{5}^{*}=3, d_{6}^{*}=1, d_{7}^{*}=2 .
$$

The corresponding value of objective function is 6.48.

The individual optimum values of Model 1 are $\xi=(64.47,-0.9994)$. Therefore, the optimum allocation under the Weighted Tchebycheff Technique of SNLPP (31) is obtained as

$$
d_{1}^{*}=1, d_{2}^{*}=3, d_{3}^{*}=1, d_{4}^{*}=2, d_{5}^{*}=1, d_{6}^{*}=1, d_{7}^{*}=1 .
$$

The corresponding value of objective function is 0.01306 .

In the same manner, we obtained the individual optimum values for Model 2 are $\xi=(277.57,-0.9989)$. Therefore, the optimum allocation under the Weighted Tchebycheff Technique of SNLPP (32) is obtained as

$$
d_{1}^{*}=1, d_{2}^{*}=2, d_{3}^{*}=0, d_{4}^{*}=2, d_{5}^{*}=2, d_{6}^{*}=0, d_{7}^{*}=1 .
$$

The corresponding value of objective function is 0.00299 .

The optimum allocations obtained corresponding to the various Bi-criteria models are summarized as given below in Table 2.

\section{Conclusions}

The multi-objective problem of allocation of repairable and replaceable components becomes complicated because an allocation that is optimal for one objective is usually far from optimal for other objectives. In such situations, we need a compromise criterion that gives an allocation which is optimum for all objectives in some sense. This paper is an attempt to utilize weighted Tchebycheff approach to the solution of optimum compromise allocation of repairable and replaceable components in a system.

The allocation problem of repairable and replaceable components for a parallel-series system considered as a Bi-objective stochastic optimization problem and discussed the four different situations. In the first situation, the reliabilities of Groups $\mathrm{X}$ and $\mathrm{Y}$ are considered as two different objectives. While in the next three situations,

Table 2. Optimum allocation of replaceable and repairable components under various $\mathrm{Bi}$-criteria models.

\begin{tabular}{ccccccccc}
\hline $\begin{array}{c}\text { S. } \\
\text { No. }\end{array}$ & $\begin{array}{c}\text { Bi-criteria } \\
\text { models }\end{array}$ & $d_{1}$ & $d_{2}$ & $d_{3}$ & $d_{4}$ & $d_{5}$ & $d_{6}$ & $d_{7}$ \\
\hline 1 & $-\operatorname{Min}\left\{R_{1}, R_{2}\right\}$ & 2 & 3 & 0 & 2 & 1 & 0 & 2 \\
2 & $\operatorname{Min}\{T, C\}$ & 2 & 1 & 0 & 2 & 1 & 0 & 3 \\
3 & $\operatorname{Min}\left\{C,-R_{1}\right\}$ & 1 & 2 & 0 & 2 & 2 & 0 & 1 \\
4 & $\operatorname{Min}\left\{T,-R_{2}\right\}$ & 1 & 3 & 1 & 2 & 1 & 1 & 1 \\
\hline
\end{tabular}

the maintenance cost and time, maintenance cost and Group X subsystem reliabilities, maintenance time and Group Y subsystem reliabilities respectively are considered as two different objectives. Selective maintenance policy is used to select the repairable and replaceable components.

An equivalent deterministic model of these Bi-objective stochastic optimization problems is established by using Chance Constrained programming method. The following four different stochastic problems are then solved by using the Bi-criteria optimization technique, weighted Tchebycheff. The weighted Tchebycheff technique provides compromise allocations of repairable and replaceable components which are optimum for both the objectives function (see Table 2). Since a compromise criteria differ from method to method, so comparison can not be made.

\section{REFERENCES}

[1] I. Ali, M. F. Khan, Y. S. Raghav and A. Bari, “Allocating Repairable and Replaceable Components for a System Availability Using Selective Maintenance with Probabilistic Constraints," American Journal of Operations Research, Vol. 1, No. 3, 2011, pp. 147-154. doi:10.4236/ajor.2011.13016

[2] W. F. Rice, C. R. Cassady and J. A. Nachlas, "Optimal Maintenance Plans under Limited Maintenance Time,” Proceedings of the 7th Industrial Engineering Research Conference, Banff, 9-10 May 1998.

[3] C. R. Cassady, E. A. Pohl and J. Song, "Managing Availability Improvement Efforts with Importance Measures and Optimization," IMA Journal of Management Mathematics, Vol. 15, No. 2, 2004, pp. 161-174. doi:10.1093/imaman/15.2.161

[4] R. Rajagopalan and C. R. Cassady, "An Improved Selective Maintenance Approach,” Journal of Quality in Maintenance Engineering, Vol. 12, No. 2, 2006, pp. 172-185. doi:10.1108/13552510610667183

[5] K. Schneider, L. M. Maillart, C. R. Cassady and C. Rainwater, "Selective Maintenance Decision-Making over Extended Planning Horizons," IEEE Transactions on Reliability, Vol. 58, No. 3, 2009, pp. 462-469. doi:10.1109/TR.2009.2026689

[6] I. M. Iyoob, C. R. Cassady and E. A. Pohl., "Establishing Maintenance Resource Levels Using Selective Maintenance," The Engineering Economist, Vol. 51, No. 2, 2006, pp. 99-114. doi:10.1080/00137910600695627

[7] I. Ali, Y. S. Raghav and A. Bari, “Allocating Repairable and Replaceable Components for a System Availability Using Selective Maintenance: An Integer Solution,” Safety and Reliability Society, Vol. 31, No. 2, 2011, pp. 9-18.

[8] I. Ali, Y. S. Raghav and A. Bari, "Integer Goal Programming Approach for Finding a Compromise Allocation of Repairable Components,” International Journal of Engineering Science and Technology, Vol. 3, No. 6, 2011, pp. 184-195. 
[9] I. Ali, Y. S. Raghav and A. Bari, "Compromise Allocation in Multivariate Stratified Surveys with Stochastic Quadratic Cost Function,” Journal of Statistical Computation and Simulation, 2011. doi:10.1080/00949655.2011.643890

[10] I. Ali, M. F. Khan, Y. S. Raghav and A. Bari, "Selective Maintenance in System Reliability with Random Costs of Repairing and Replacing the Components," Communications in Statistics-Simulation and Computation, 2012. http://www.tandfonline.com/doi/abs/10.1080/03610918.2 012

[11] M. F. Khan and I. Ali, "Allocation Problem of Repairable and Replaceable Components for a System Reliability using Selective Maintenance: An Integer Solution,” International Journal of Scientific and Engineering Research, Vol. 3, No. 5, 2012, pp. 1-4.

[12] S. S. Rao, "Optimization Theory and Applications,” Wiley Eastern Limited, New Delhi, 1979.
[13] V. J. Bowman Jr., "On the Relationship of the Tchebycheff Norm and the Efficient Frontier of Multiple-Criteria Objectives,” In: H. Thiriez and S. Zionts, Eds., "Multiple Criteria Decision Making," Lecture Notes in Economics and Mathematical Systems, Springer-Verlag, Berlin, 1976, pp. 76-85.

[14] K. M. Miettinen, "Non Linear Multiobjective Optimization,” Kluwer Academic Publishers, Boston, 1999.

[15] A. P. Wierzbicki, "On the Completeness and Constructiveness of Parametric Characterizations to Vector Optimization Problem," Operations-Research-Spektrum, Vol. 8, No. 2, 1986, pp. 73-87. doi:10.1007/BF01719738

[16] M. T. Khasawneh, S. R. Bowling, S. Kaewkuekool and B. R. Cho, "A Cost-Effective Strength-Stress Reliability Modeling and Optimization in Engineering Design," Proceeding of the Industrial Engineering Research Conference, Orlando, 19-21 May 2002. 